\title{
Sea lice infestations on juvenile chum and pink salmon in the Broughton Archipelago, Canada, from 2003 to 2012
}

\author{
Thitiwan Patanasatienkul ${ }^{1, *}$, Javier Sanchez ${ }^{1}$, Erin E. Rees $^{1}$, Martin Krkošek ${ }^{2,3}$, \\ Simon R. M. Jones ${ }^{4}$, Crawford W. Revie ${ }^{1}$
}

\author{
${ }^{1}$ Atlantic Veterinary College, University of Prince Edward Island, 550 University Ave, Charlottetown, Prince Edward Island C1A 4P3, \\ Canada \\ ${ }^{2}$ Department of Ecology and Evolutionary Biology, University of Toronto, 25 Willcocks Street, Toronto, Ontario M5S 3B2, Canada \\ ${ }^{3}$ Department of Zoology, University of Otago, 340 Great King Street, Dunedin 9016, New Zealand \\ ${ }^{4}$ Pacific Biological Station, Fisheries and Oceans Canada, 3190 Hammond Bay Road, Nanaimo, British Columbia V9T 6N7, Canada
}

\begin{abstract}
Juvenile pink salmon Oncorhynchus gorbuscha and chum salmon O. keta were sampled by beach or purse seine to assess levels of sea lice infestation in the Knight Inlet and Broughton Archipelago regions of coastal British Columbia, Canada, during the months of March to July from 2003 to 2012. Beach seine data were analyzed for sea lice infestation that was described in terms of prevalence, abundance, intensity, and intensity per unit length. The median annual prevalence for chum was 30\%, ranging from $14 \%$ (in 2008 and 2009) to $73 \%$ (in 2004), while for pink salmon, the median was $27 \%$ and ranged from $10 \%$ (in 2011) to $68 \%$ (in 2004). Annual abundance varied from 0.2 to 5 sea lice per fish with a median of 0.47 for chum and from 0.1 to 3 lice (median 0.42 ) for pink salmon. Annual infestation followed broadly similar trends for both chum and pink salmon. However, the abundance and intensity of Lepeophtheirus salmonis and Caligus clemensi, the 2 main sea lice species of interest, were significantly greater on chum than on pink salmon in around half of the years studied. Logistic regression with random effect was used to model prevalence of sea lice infestation for the combined beach and purse seine data. The model suggested inter-annual variation as well as a spatial clustering effect on the prevalence of sea lice infestation in both chum and pink salmon. Fish length had an effect on prevalence, although the nature of this effect differed according to host species.
\end{abstract}

KEY WORDS: Sea lice - Lepeophtheirus salmonis - Caligus clemensi - Oncorhynchus keta · Oncorhynchus gorbuscha · Broughton Archipelago · Epidemiology

Resale or republication not permitted without written consent of the publisher

\section{INTRODUCTION}

Pink and chum salmon are the most abundant species of anadromous salmon in the North Pacific Ocean (Noakes \& Beamish 2011). Juveniles belonging to these species are similar in that they enter estuarine habitats soon after emergence at mean weights of $<1 \mathrm{~g}$. Juvenile pink and chum salmon disperse into deeper coastal waters following the initial period of adaptation to saltwater that occurs in nearshore or estuarine habitats (see reviews by Heard
1991 and Salo 1991). Subsequent rapid growth in the presence of suitable forage in the marine environment is typical for juvenile pink and chum salmon (Heard 1991, Moss et al. 2009). The tendency of these early juvenile salmon to occupy relatively shallow nearshore habitat has been associated with an increased risk of exposure to sea lice (Morton \& Williams 2003, Jones \& Hargreaves 2007, Gottesfeld et al. 2009).

Sea lice are parasitic copepods of the family Caligidae which infest both wild and farmed salmonids 
(Boxaspen 2006, Wagner et al. 2008). Lepeophtheirus salmonis and Caligus spp. are the most commonly reported sea lice species on salmonids (Morton \& Williams 2003, Wertheimer et al. 2003, Jones \& Nemec 2004, Beamish et al. 2005, Krkošek et al. 2005b, Boxaspen 2006, Rolston \& Proctor 2003, Price et al. 2010, 2011). Sea lice feed on the host skin, mucus, and blood, which can cause skin erosion and induce secondary infection (Mustafa 1997). Large numbers of mobile L. salmonis can cause host morbidity and mortality (Pike \& Wadsworth 1999, Tully \& Nolan 2002, Johnson \& Fast 2004). L. salmonis has been associated with decreases in swimming performance and post-swim body ion concentrations that are particularly evident in the smallest juvenile pink salmon (Nendick et al. 2011). Lice attachment also causes a generalized stress response mediated through cortisol release resulting in immune function suppression (Wagner et al. 2008). Sea lice infestation has been a problem for aquaculture producers in many countries including Norway, Chile, Scotland, Ireland, and Canada (Heuch et al. 2003, Jones \& Nemec 2004, Hamilton-West et al. 2012, Jackson et al. 2012). The issue of sea lice infestation in Pacific wild salmon populations has been a focus of attention over the past decade; several surveys and monitoring programs have been developed to evaluate sea lice infestation on wild salmon at various locations on the west coast of Canada (Jones \& Nemec 2004, Beamish et al. 2005, Krkošek et al. 2005b, 2006, Jones et al. 2006, Jones \& Hargreaves 2007). Some studies have addressed the issue of louse infestation in wild salmon associated with the open sea-cage aquaculture (Jacobsen \& Gaard 1997, Krkošek et al. 2005a, 2007a, 2011b, Morton et al. 2005, 2008, Marty et al. 2010, Price et al. 2010, 2011, Saksida et al. 2011). Jones \& Nemec (2004) suggested that spatial aggregation may be an important dimension in understanding the impact on sea lice infestations of wild salmon. Some inter-annual variability has been reported in previous studies (Jones et al. 2006, Jones \& Hargreaves 2007, 2009, Krkošek et al. 2007b, Saksida et al. 2011); however, no studies have reported these trends over a full decade.

Two different sampling protocols were used in the major studies carried out in the Broughton Archipelago: live (Krkošek et al. 2005a,b, 2006, 2007a, 2011b) and lethal (Jones \& Nemec 2004, Morton et al. 2005, Jones et al. 2006, Jones \& Hargreaves 2009, Saksida et al. 2011) methods. The live sampling protocol was unable to identify the species or developmental stage for sea lice in early life stages, while with the lethal method the fish were collected and sent to a labora- tory, allowing for a detailed evaluation of any lice present. All of the samples examined under the live protocol were caught by beach seine, whereas those assessed using the lethal protocol were caught either by beach or purse seine. Patterns of lice infestation reported from these studies in the past appeared to be somewhat different from one another, and it was assumed that variation in sampling protocols and regions targeted were at least partially responsible for this variability. In 2010, a collaborative research program, the Broughton Archipelago Monitoring Program (BAMP), was developed with involvement of the salmon farming companies operating in the Broughton Archipelago, Fisheries and Oceans Canada (DFO), university researchers, and the Coastal Alliance for Aquaculture Reform (CAAR), with the objective of better understanding sea lice levels and their dynamics on juvenile wild pink and chum salmon in the Broughton Archipelago. As part of the BAMP initiative (www.bamp.ca), historical data were pooled to create a unified database, while a standard sampling protocol was also developed and used starting in 2010 through to the present.

The objectives of this study were to describe the prevalence, abundance, and intensity of sea lice infestation on out-migrating juvenile pink and chum salmon in the Broughton Archipelago during sampling months (March to July) from 2003 to 2012 using beach seine data and to model factors, such as fish species, fish length, month, and gear type used for sampling, that may be associated with the interannual trends in prevalence of sea lice infestation using the combined beach and purse seine data.

\section{MATERIALS AND METHODS}

\section{Study area}

The study area is located in the Knight Inlet and Broughton Archipelago regions of coastal British Columbia, Canada. There are 160 sampling sites which were repeatedly sampled by the Krkošek or DFO teams during 2003 to 2009 (Fig. 1). The sample design for the Krkošek team was designed to intensively study infestations on juvenile salmon as they migrate through Knight Inlet and Tribune Channel. The sample design for DFO provided a broader representation of locations and habitats across the study area. In 2010 and 2011, the BAMP initiative reduced the number of sites to 98 , and further to 79 sites in 2012, by progressively eliminating sites at which few, or no, fish had been caught in previous years. 


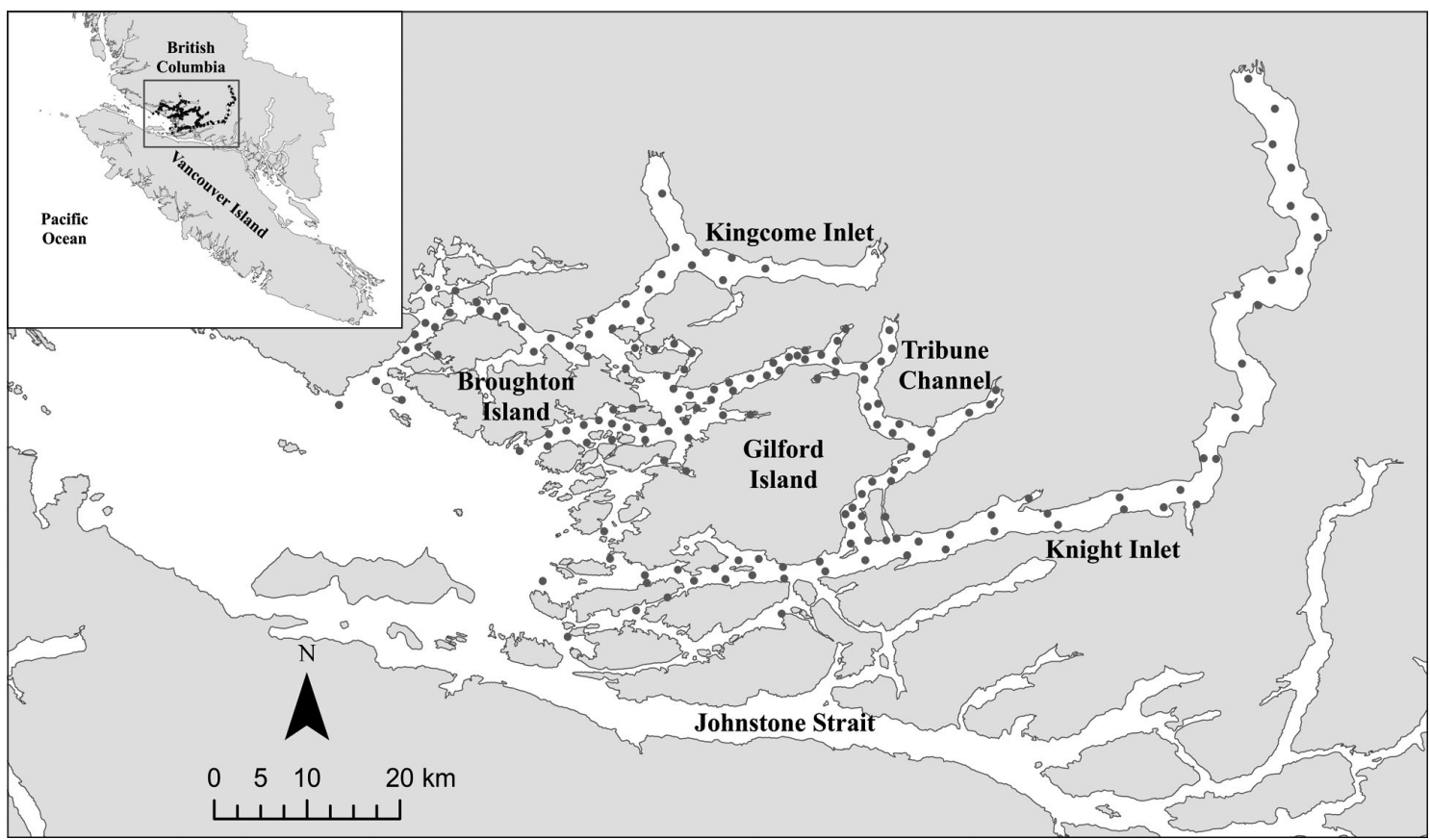

Fig. 1. Broughton Archipelago, showing the study area and median coordinates of sampling sites during 2003 and 2012

\section{Sampling}

Sampling was carried out using 1 of 3 different protocols: the non-lethal sampling method by the Krkošek team described by Krkošek et al. (2005b), the lethal sampling method used by DFO (Jones \& Nemec 2004, Jones et al. 2006), and the BAMP protocol (www. bamp.ca), which merges the spatial distribution of sampling effort between the 2 previous programs and which uses lethal lab-based analysis of lice on fish. Data collection took place weekly, biweekly, or monthly during the sampling period March to July over the years 2003 to 2012. Table 1 describes the sampling intervals for each type of collection by year. Two fishing gear types, beach and purse seines, were used to collect samples. Purse seine was used only by DFO from 2003 to 2009. The geographical coordinates for every sampling site were collected. Typically, at each site, a maximum of 100 specimens of each species (Krkošek team) or 30 of each species (DFO and BAMP) were randomly selected from the sample. Each fish was measured for fork length $(\mathrm{mm})$ and wet weight $(\mathrm{g})$. Weight was not recorded by the Krkošek team due to difficulty weighing live fish. Lice count, developmental stage (copepodite, chalimus, pre-adult, or adult), species (Caligus clemensi, Lepeophtheirus salmonis, or not identified to species), and gender (motile stages) data were also collected when the lethal sampling protocol was used. In the case of the non-lethal sampling method, lice were categorized to broad developmental stage, but only motile lice were identified to species.

\section{Statistical analyses}

A total of 166316 fish were available for sea lice infestation analysis over the study period. Data quality checking for missing or biologically implausible fish lengths was carried out. All lice were categorized as 'non-motile' (copepodite or chalimus stages) or 'motile' (preadult or adult stages). A more detailed assessment of specific developmental stages was not possible given that some lice were not classified to this level. Lice that were not identified by species were classified as 'not identified to species.'

Table 1. Sampling intervals by collector and year. DFO: Fisheries and Oceans Canada; BAMP: Broughton Archipelago Monitoring Program; W: weekly; BW: biweekly; M: monthly; -: no data

\begin{tabular}{lccccccccccc} 
Collector & 2003 & 2004 & 2005 & 2006 & 2007 & 2008 & 2009 & 2010 & 2011 & 2012 \\
\hline Krkošek team & M & M & - & M & M & M & M & - & - & - \\
DFO & W & BW & M & M & M & M & M & - & - & - \\
BAMP & - & - & - & - & - & - & - & M & M & M \\
\hline
\end{tabular}


Means and 95\% confidence intervals for fork length and weight of chum and pink salmon were calculated. The total numbers of salmon used to calculate mean weights differed from those used in the estimation of mean length, as weight values were missing for 43750 chum and 40428 pink salmon. Fish with at least 1 sea louse of any species and any developmental stage were classified as infested. Louse infestation of each fish species was expressed in terms of prevalence (proportion of fish infested), mean louse abundance (lice per fish), and mean louse intensity (lice per infested fish) after Bush et al. (1997), and mean lice intensity per fish length (lice $\mathrm{mm}^{-1}$ of infested fish). We used a robust standard error to adjust for clustering of sampling sites when estimating the $95 \%$ confidence intervals associated with lice infestation (for details on robust standard error, see Dohoo et al. 2009).

To determine whether to combine beach and purse data for descriptive statistics, a comparison of fish length between samples caught using beach and purse nets was made using a paired $t$-test for all locations at which both gear types were used on the same day. As fish size between the 2 gear types was different (see 'Results'), and there were more data across the years from beach seine sampling, we restricted our analyses concerning lice infestation to these data.

The difference in proportion of Lepeophtheirus salmonis and Caligus clemensi was computed with the total number of lice which were identified to species in a given year as a denominator. A 0 difference represents an equal proportion of lice from each species. A positive value represents a higher proportion of $L$. salmonis, while a negative value represents a higher proportion of $C$. clemensi. The proportion of all lice that were identified to species in a given year was also calculated. The results are presented using a modified forest plot with weightings derived from the proportions of lice identified to species.

Multivariable logistic regression analysis was used to assess factors affecting levels of lice infestation. Both beach and purse data were included in the model. The presence or absence of sea lice (all species) on fish was used as the outcome variable. Year, month, fish length, fish species, and gear type were included as fixed effect predictors, and sampling site as a random effect. The assumptions for the logistic regression model were assessed (Dohoo et al. 2009). As the assumption for linearity between fish length and the outcome variable was not met, the quadratic term of this variable was added and checked for statistical significance of the term. An interaction between fish species and fish length was also included in the model to account for potential differences in growth characteristics between species (Moss et al. 2009). Akaike's information criterion (AIC) was used to assess the fit of alternate models (Burnham 2002).

\section{RESULTS}

\section{Sampling instances and sampled fish}

A total of 7396 sampling instances were carried out in the Broughton Archipelago area from 2003 to 2012 during mostly monthly surveys (Table 1), providing a total of 166194 sampled fish (122 fish were excluded from the analyses due to missing data) from the combined beach and purse seine data. Typically, 4 to 5 monthly samples were collected between March and July, with some variation among years (Table 2). The number of sampling instances per year varied from 236 to 1514 (Table 2). Over the course of the whole study, the average $( \pm \mathrm{SD})$ number of fish assessed per

Table 2. Oncorhynchus keta and O. gorbuscha. Number of sampling instances, and number of wild chum and pink salmon sampled by beach or purse seine assessed per year by sampling month in the Broughton Archipelago from 2003 to 2012

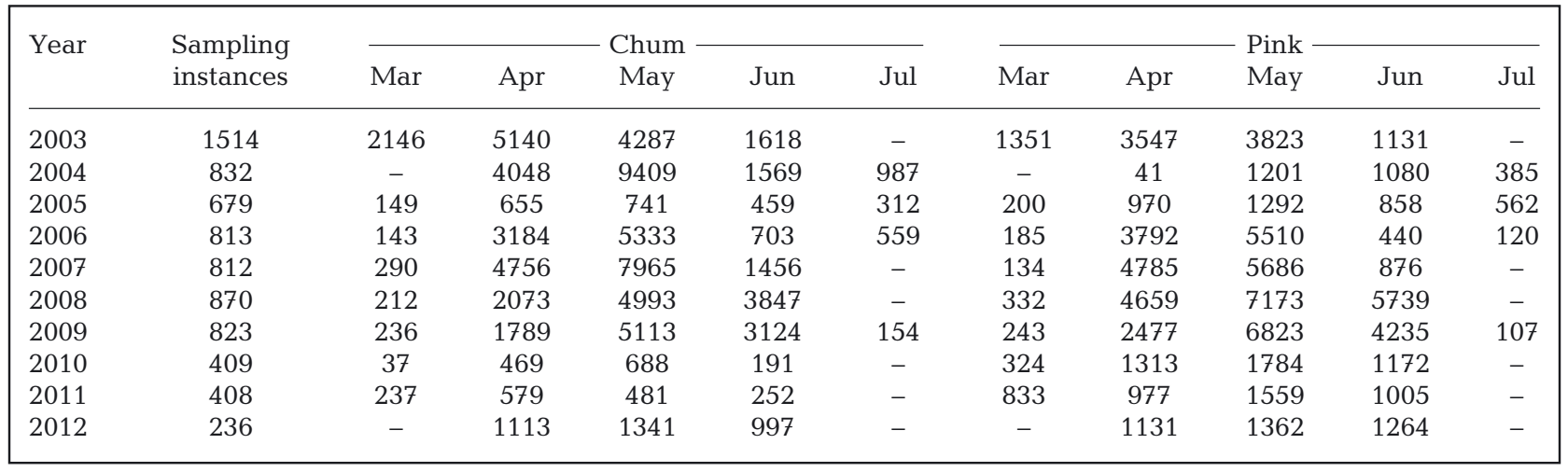


sampling instance was $11( \pm 19.1)$ chum and $12( \pm 19.4)$ pink salmon. In some years (e.g. 2004) chum salmon dominated the samples, while in other years, especially from 2008 onwards, pink salmon were the dominant species sampled.

A summary of the physical characteristics of the salmon sampled can be found in Table S1 in the Supplement (www.int-res.com/articles/suppl/d105 p149_supp.pdf). A paired comparison indicated that fish caught using a purse net were significantly longer than those caught by a beach seine at the same time/location, with mean differences in length of $15.4 \mathrm{~mm}$ (95\% CI: 13.8-17.0) and $7.8 \mathrm{~mm} \mathrm{(95 \%} \mathrm{CI:} \mathrm{6.6-9.1)} \mathrm{for} \mathrm{chum}$ $(\mathrm{n}=356)$ and pink $(\mathrm{n}=265)$ salmon, respectively. In the case of beach-seined fish, far more data were reported for length than weight (Table S1). As a consequence, we assessed only length as a size metric in our analyses. The breakdown of length estimates by year shown in Fig. 2 indicates a similar finding with additional information on annual variation. As a result of the significant association of gear type and fish size, all analyses for lice infestation include only the beach-seined fish with the exception of a multivariable model that includes both beach and purse seine data.

A similar pattern of apparent growth in fish size over the season is shown for chum and pink salmon in Fig. 3. For pink salmon (Fig. 3b), there appear to be few clear differences among the years. In the case of chum salmon (Fig. 3a), it is interesting to note that in one year (2009) apparent growth was consistently slower while in another (2005) fish exhibited much faster growth.

\section{Sea lice infestation}

Table 3 provides a summary of the overall sea lice infestation in terms of mean annual prevalence, abundance, and intensity for chum and

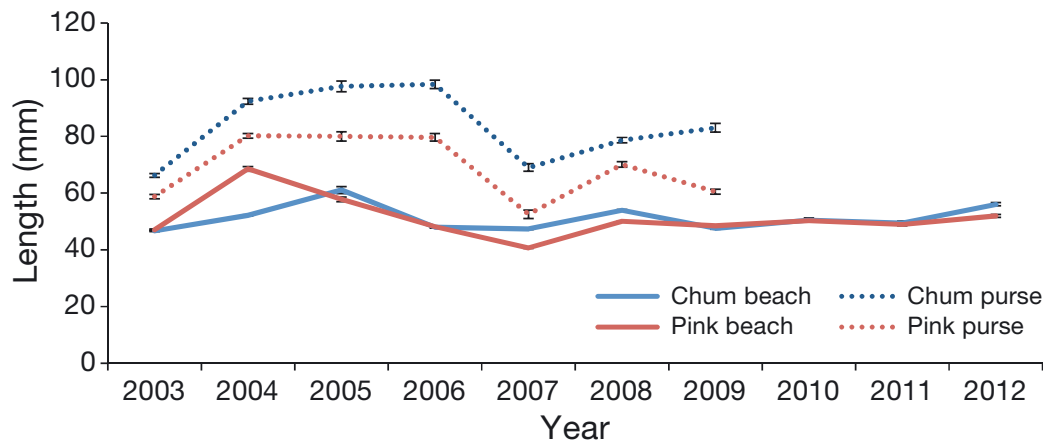

Fig. 2. Oncorhynchus keta and O. gorbuscha. Average length with $95 \%$ confidence intervals for wild chum and pink salmon in the Broughton Archipelago assessed during the sampling months (March to July) from 2003 to 2012 by gear type

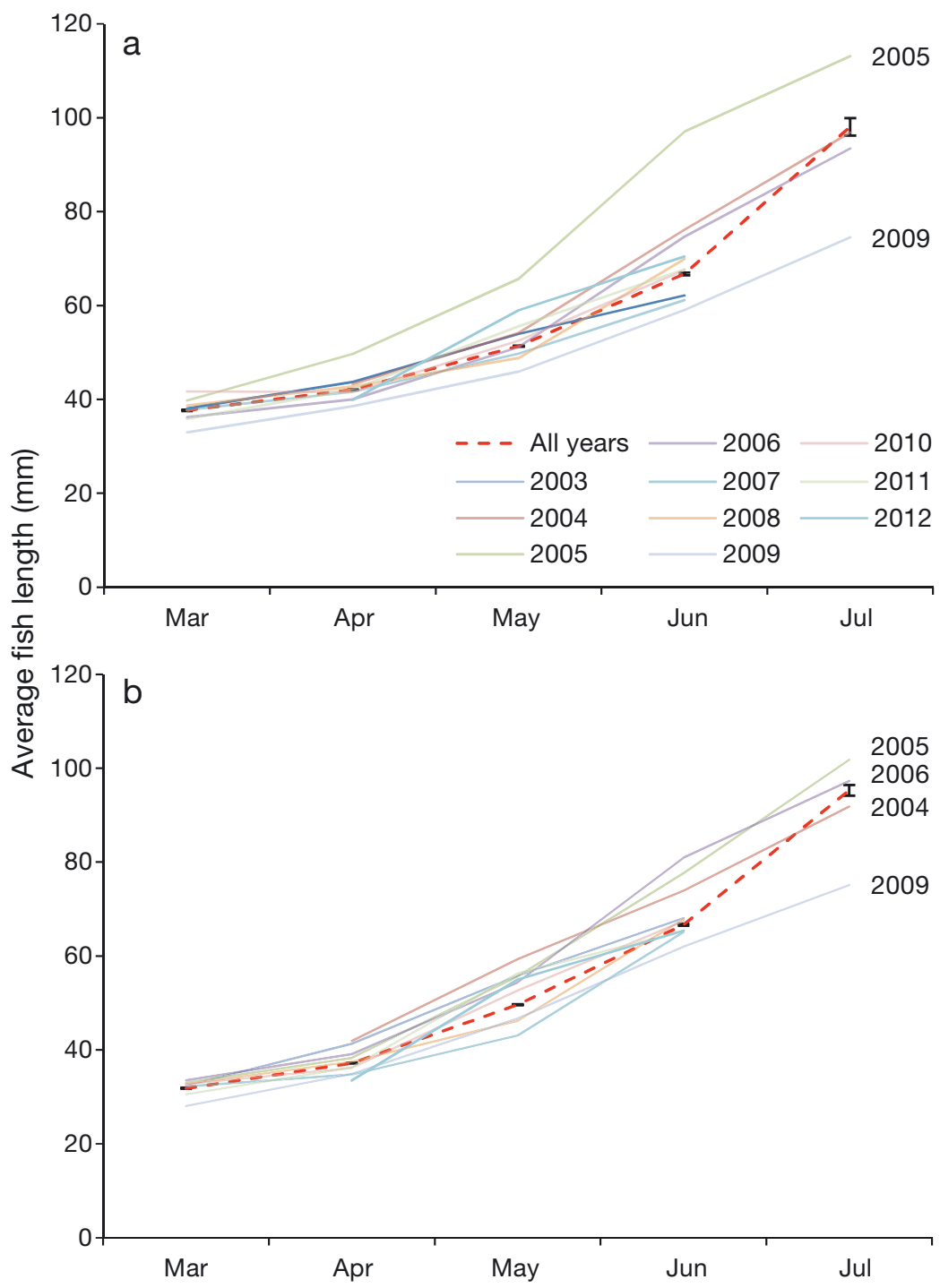

Fig. 3. Oncorhynchus keta and O. gorbuscha. Monthly average length of wild (a) chum and (b) pink salmon in the Broughton Archipelago sampled by beach seine from 2003 to 2012 
Table 3. Lepeophtheirus salmonis and Caligus clemensi infesting Oncorhynchus keta and O. gorbuscha. Mean and 95\% confidence intervals adjusted for site clustering for prevalence of lice infestation, lice abundance, and lice intensity in wild chum and pink salmon sampled by beach seine in the Broughton Archipelago from 2003 to 2012

\begin{tabular}{|c|c|c|c|c|c|c|c|c|}
\hline \multirow{2}{*}{ Year } & \multicolumn{4}{|c|}{ Chum } & \multicolumn{4}{|c|}{ - Pink } \\
\hline & $\mathrm{n}$ & Prevalence & Abundance & Intensity & $\mathrm{n}$ & Prevalence & Abundance & Intensity \\
\hline 2003 & 10127 & $0.32[0.26,0.39]$ & $0.70[0.52,0.89]$ & $2.18[1.96,2.40]$ & 7518 & $0.32[0.27,0.38]$ & $0.53[0.43,0.64]$ & $1.65[1.54,1.76]$ \\
\hline 2004 & 13086 & $0.73[0.66,0.80]$ & $5.05[4.14,5.96]$ & $6.90[6.02,7.78]$ & 1308 & $0.68[0.64,0.72]$ & $3.05[2.40,3.69]$ & $4.48[3.69,5.26]$ \\
\hline 2005 & 1299 & $0.38[0.29,0.47]$ & $1.15[0.76,1.54]$ & $3.03[2.57,3.50]$ & 2723 & $0.32[0.27,0.37]$ & $0.64[0.51,0.78]$ & $2.01[1.81,2.22]$ \\
\hline 2006 & 8701 & $0.27[0.23,0.32]$ & $0.41[0.33,0.49]$ & $1.50[1.42,1.59]$ & 8994 & $0.31[0.26,0.37]$ & $0.53[0.41,0.65]$ & $1.68[1.56,1.81]$ \\
\hline 2007 & 13229 & $0.41[0.37,0.45]$ & $0.81[0.69,0.93]$ & $1.99[1.87,2.10]$ & 10711 & $0.34[0.30,0.37]$ & $0.58[0.50,0.65]$ & $1.72[1.66,1.78]$ \\
\hline 2008 & 9530 & $0.14[0.11,0.17]$ & $0.20[0.14,0.27]$ & $1.44[1.22,1.65]$ & 16390 & $0.13[0.10,0.16]$ & $0.17[0.12,0.23]$ & $1.35[1.22,1.47]$ \\
\hline 2009 & 9262 & $0.14[0.12,0.16]$ & $0.17[0.14,0.21]$ & $1.26[1.19,1.34]$ & 12170 & $0.12[0.10,0.14]$ & $0.14[0.12,0.17]$ & $1.18[1.13,1.23]$ \\
\hline 2010 & 1384 & $0.30[0.25,0.35]$ & $0.46[0.37,0.56]$ & $1.53[1.40,1.66]$ & 4591 & $0.23[0.21,0.26]$ & $0.32[0.28,0.36]$ & $1.38[1.33,1.42]$ \\
\hline 2011 & 1548 & $0.16[0.12,0.20]$ & $0.23[0.16,0.31]$ & $1.46[1.34,1.58]$ & 4374 & $0.10[0.08,0.12]$ & $0.12[0.10,0.15]$ & $1.22[1.18,1.27]$ \\
\hline 2012 & 3450 & $0.30[0.26,0.34]$ & $0.48[0.40,0.55]$ & $1.58[1.46,1.70]$ & 3755 & $0.18[0.16,0.20]$ & $0.24[0.20,0.28]$ & $1.33[1.27,1.39]$ \\
\hline
\end{tabular}

pink salmon caught by beach seine. Annual sea lice abundance varied from as low as 0.17 (2009) to as high as 5.05 per fish (2004) for chum, and from 0.12 (2011) to 3.05 per fish (2004) for pink salmon. It should be noted, however, that 2004 appears to be very much the outlier in this set of observations, with only one other annual average (chum in 2005) being $>1$ louse per fish. Approximately half of the years assessed have a mean abundance $<0.5$ lice per chum or per pink. There are no data from 2010 to 2012 in the purse seine samples (Table S2 in the Supplement) due to the modification in sampling protocol. Sea lice abundance for the fish sampled by purse net ranged from 0.07 (2008) to 5.78 per fish (2004) for chum, and from 0.17 (2009) to 0.69 per fish (2004) for pink salmon.

The annual prevalence estimates and their 95\% CIs are illustrated in Fig. 4. The median prevalence was around $30 \%$, although in many of the latter years, this value fell to below $20 \%$, with 2004 once again proving the exception with a prevalence of around $70 \%$. The graphical summary indicates that the prevalence of sea lice on both salmon species tended to show similar trends over time. Indeed, the CIs for the prevalence estimates of sea lice on chum and pink salmon overlapped in every year apart from 2012, suggesting that significant differences are not common. In contrast, it can be seen from Table 3 that when considering either the abundance or intensity estimates for sea lice on chum, these were significantly higher than those on pink salmon in around half of the years (i.e. $95 \%$ CIs show no overlap).

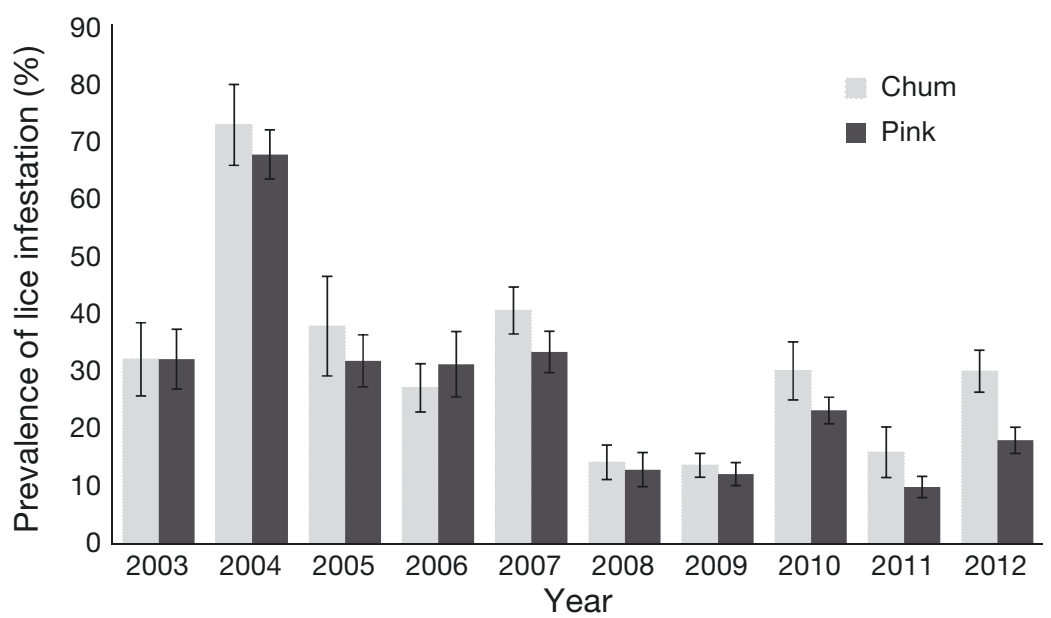

Fig. 4. Oncorhynchus keta and O. gorbuscha. Yearly prevalence and $95 \%$ confidence intervals adjusted for site clustering of lice infestation in wild chum 政 2003 to 2012

\section{Sea lice species}

A total of 150060 sea lice on beach- and purseseined fish were assessed over the course of the study. Around 38\% were identified as Lepeophtheirus salmonis and $9 \%$ as Caligus clemensi, while the remainder were not identified to species. Variation in the proportion of lice species observed and the percent classified for beach seine data are detailed per year in Table S3 in the Supplement. From this point, the analyses focused on beach seine data. Fig. 5 summarizes the difference in proportions of lice identified as either L. salmonis or $C$. clemensi from all lice which were identified to species in a given year. In the early years, pre-2009, $L$. salmonis was the dominant species with between 48 and $84 \%$ more lice than those recorded as $C$. 


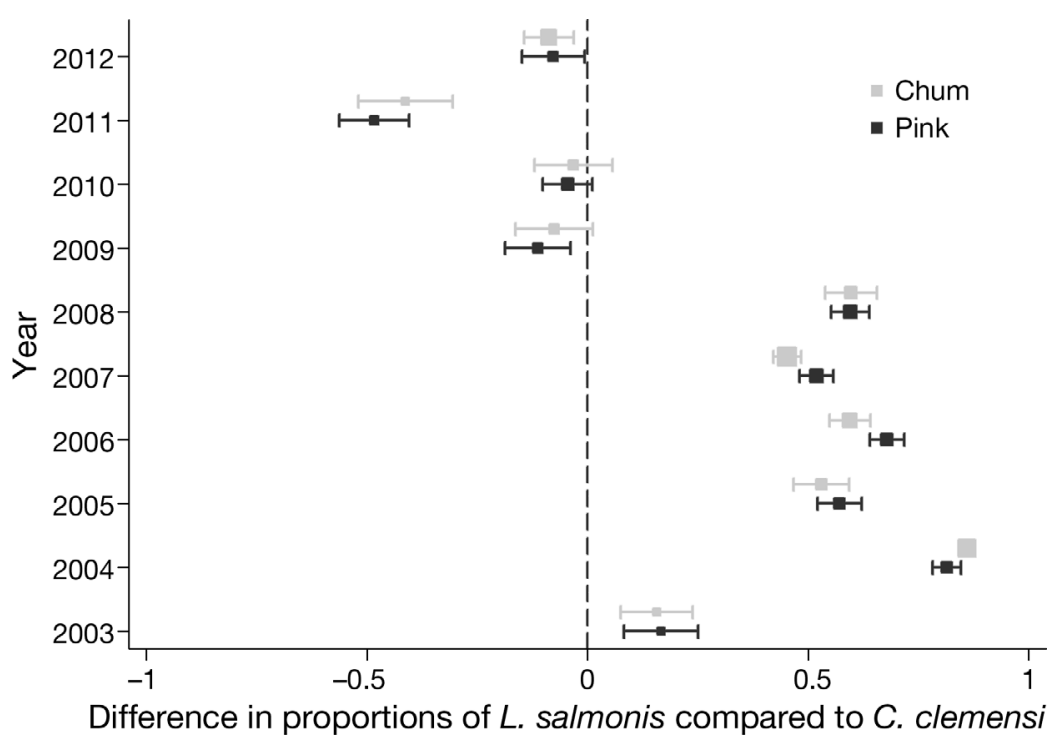

Fig. 5. Lepeophtheirus salmonis and Caligus clemensi infesting Oncorhynchus keta and O. gorbuscha. Average difference in proportion of L. salmonis compared to C. clemensi for all lice that were identified to species, on wild chum and pink salmon sampled by beach seine in the Broughton Archipelago, assessed from 2003 to 2012 . The area of the box is proportional to the number of infested fish where all lice were identified to species. Negative values refer to a higher proportion of $C$. clemensi while positive values indicate a higher proportion of L. salmonis

clemensi. The exception was 2003, when the proportions were closer, with difference values of just over $15 \%$. However, these were based on a small sample set (only $13 \%$ of the 11133 reported in that year), so the result should be treated with some caution. The sizes of the solid squares in Fig. 5 are proportional to the numbers of infested fish on which all lice present were identified to species in each of the years. It is interesting to note that in only one year (2011) was there clear evidence of $C$. clemensi being the dominant species observed on the salmon sampled. As can be seen, in all years, the results indicate that similar differences in the proportion of L. salmonis compared to C. clemensi were found to be present on both chum and pink salmon.

Within the annual summaries, as well as the multivariable model which follows, we chose to adopt prevalence as the key measure of infestation. Fig. 6 illustrates the strong linear relationship between prevalence and abundance when prevalence was less than $60 \%$. As expected, this relationship ceases to hold when sea lice prevalence levels reach $65 \%$ or more, as was the case in 2004. There also appears to be an outlier in the monthly data of pink salmon in March of 2005, which has an abundance value over twice the level that might be expected given a prevalence of $26 \%$.
As can be seen from Table 3, louse intensity ranged from 1.26 (2009) to 6.90 (2004) for chum and from 1.18 (2009) to 4.48 (2004) for pink salmon. Intensities broken down by month are illustrated in Fig. 7. As was noted above, 2004 exhibited a high level of prevalence (Fig. 4) which leads to qualitatively distinct patterns of infestation (Fig. 6). The inclusion of these heavily infested fish would thus have a disproportionate impact on mean intensity, and for this reason data from 2004 were excluded from the estimate of intensity per unit length. Fig. 7 shows that for all infested fish, although both chum and pink salmon started the season exhibiting no difference in lice intensity, chum exhibited a modest increase in intensity as the season developed, while the intensity of lice on pink salmon remained largely constant, at around 1.5 lice per fish. Normalizing intensity by fish length shows the decreasing trend for both fish species.

\section{Non-motile and motile lice}

The development of sea lice coincides with growth of the juvenile salmon during their residence in the study area. Sea louse development was assessed in terms of proportion of motile lice in samples taken over time. Fig. 8 shows the monthly average proportion of motile lice in comparison to all lice sampled. In general, this proportion increased as the season progressed for both fish species. However, the proportion of motile lice on pink salmon was significantly higher than for chum salmon from May onwards.

The analyses above have purposely not taken into account the species of lice which were identified as being present on the fish. As has been noted, many of the lice observed, particularly those in the non-motile stages, were not labeled according to species. The proportion of lice that were identified to species ranged from $13 \%$ in 2003 to $100 \%$ of the samples (under the BAMP protocol from 2010). However, prevalence trend analyses incorporating sea lice species, where available, resulted in similar patterns and trends (Fig. S1 in the Supplement). 


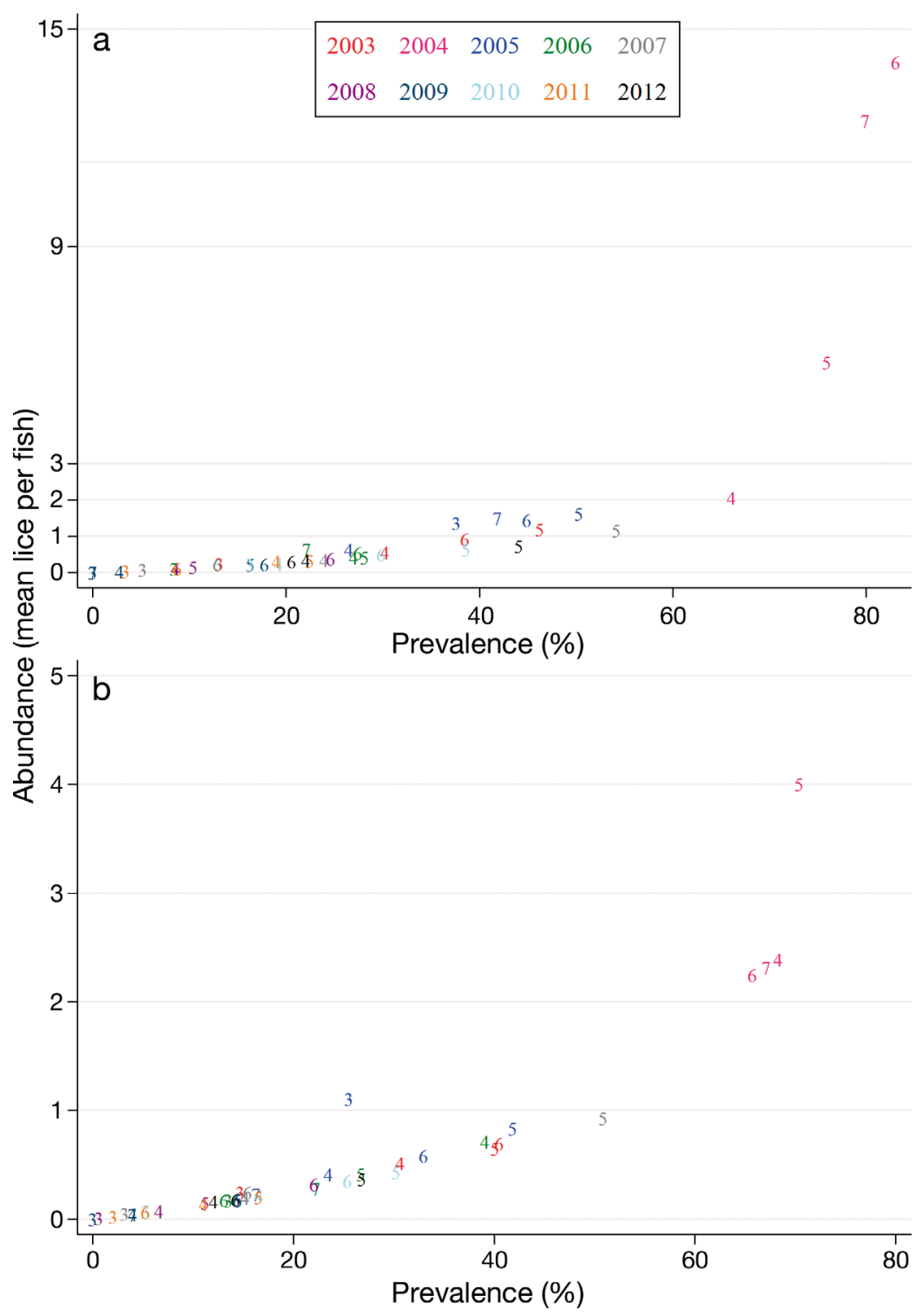

Fig. 6. Lepeophtheirus salmonis and Caligus clemensi infesting Oncorhynchus keta and O. gorbuscha. Lice infestation prevalence and lice abundance for (a) chum and (b) pink salmon sampled by beach seine in the Broughton Archipelago assessed from 2003 to 2012 with the numbers representing sampling month from March (3) to July (7); colours show years fish with a louse were around 4 times greater than in 2003. The likelihood of lice infestation was lowest in 2009, with an odds ratio of 0.16 in comparison to 2003 , or roughly 25 times lower than that seen in 2004. The likelihood of finding fish with a louse increased from March to May, which had the highest odds ratio of 2.77 , after which the likelihood decreased in the final 2 months sampled and by July was no different from the odds of having an infestation in March. Fish sampled by purse net had reduced odds of 0.6 of having lice present on them when compared to those sampled by beach seine. A significant interaction between fish species and fish length was found. In general, a quadratic relationship was found to hold between length and sea lice prevalence in that both smaller and larger fish had a reduced likelihood of infestation, while midsized fish were most likely to be infested. The interaction was due to the fact that this 'convex' relationship was more pronounced in chum than was the case for pink salmon.

Site variance was significantly different from 0, suggesting a spatial clustering effect. The model estimated that the proportion of the variance was $38 \%$ at the site level (Table 4 ). The site median odds ratio for 2 fish with identical risk factors from 2 randomly selected sites is 3.9 , suggesting that if a fish moves from one site to another site with a higher risk, its likelihood of being an infested fish will increase 3.9 times and thus that inter-site variation has a strong impact on the likelihood of infestations for individual fish.

\section{DISCUSSION}

A summary of results from the logistic model of factors associated with the presence of sea lice is given in Table 4. Year, month, gear type, fish species, and fish length were all significant factors, as was the interaction between fish species and fish length. The model shows a better fit when interaction terms were included. Diagnostic analysis provided no indication of heteroscedasticity, and modeled residuals were normally distributed. In 2004, the odds of finding a
This research summarizes findings from the longest continuous surveillance effort for sea lice in populations of juvenile wild salmon. The temporal trends in sea lice infestations were generally mirrored in the 2 fish species, though at a higher magnitude in chum. The lice were identified to species and stage-classified only when the lethal protocol was used, as a re- 


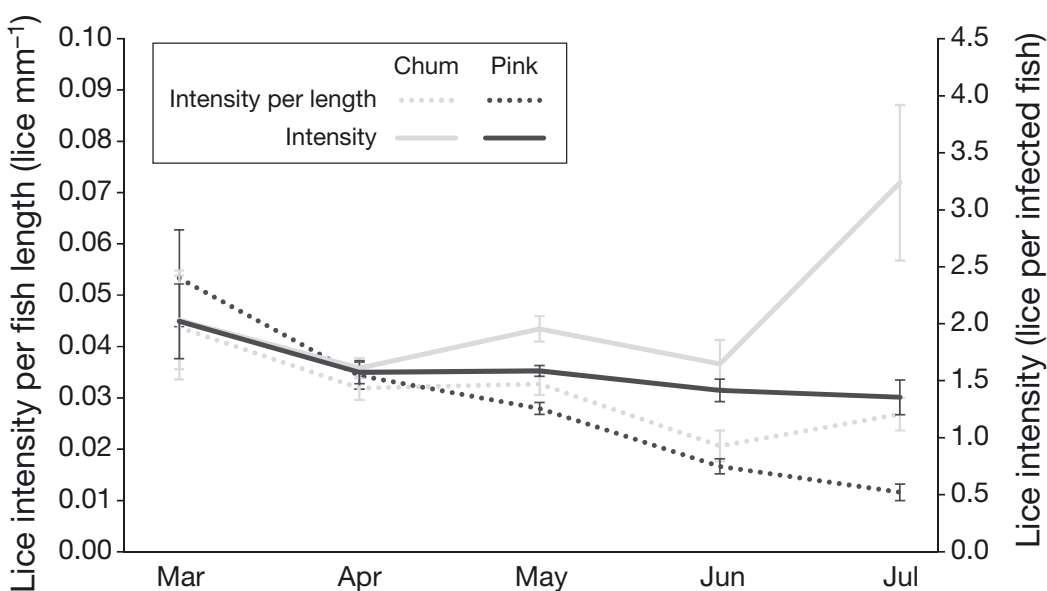

Fig. 7. Lepeophtheirus salmonis and Caligus clemensi infesting Oncorhynchus keta and O. gorbuscha. Monthly lice intensity and lice intensity per fish length (means and 95\% confidence intervals) adjusted for site clustering for wild chum and pink salmon sampled by beach seine in the Broughton Archipelago assessed in 2003 and from 2005 to 2012

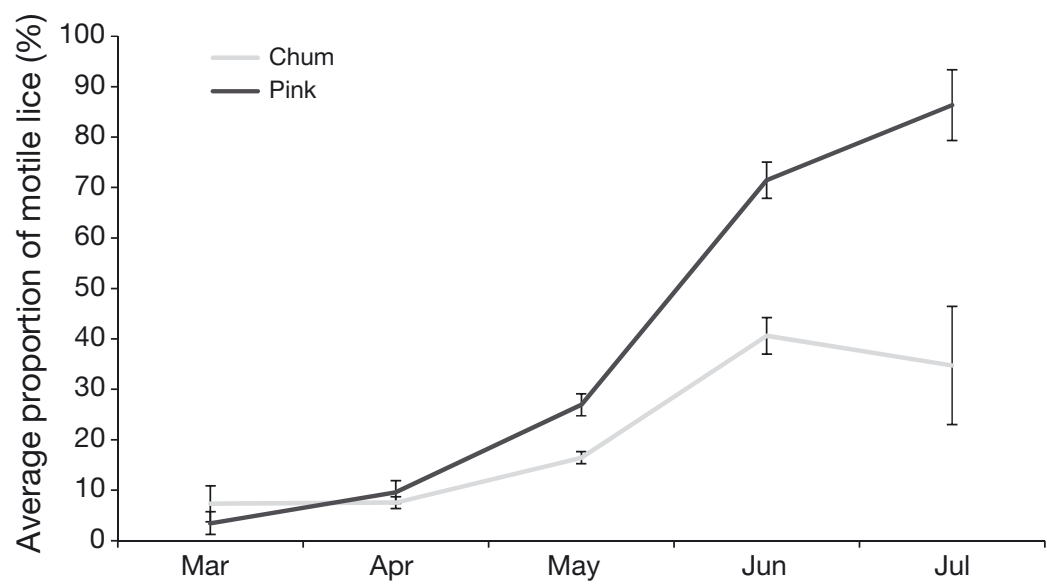

Fig. 8. Lepeophtheirus salmonis and Caligus clemensi infesting Oncorhynchus keta and O. gorbuscha. Average proportion of motile lice to all lice per fish and $95 \%$ confidence intervals adjusted for site clustering on wild chum and pink salmon sampled by beach seine in the Broughton Archipelago assessed from 2003 to 2012

sult of which most of the analysis was carried out at the coarse level by combining the 2 lice species (Lepeophtheirus salmonis and Caligus clemensi) and aggregating lice stages to motile or non-motile lice.

Incorporating beach and purse seines provided an opportunity to sample juvenile salmon over a broader range of sizes than was possible with beach seines alone. This reflects the tendency of smaller fish to remain in near-shore waters and thus more likely to be caught by beach seine, which is suitable for shallow water (Hahn et al. 2007). The association be- tween gear type and fish size reflects this behavior and agrees with the findings of several other studies (Johnsen \& Sims 1973, Sims \& Johnsen 1974, Dawley et al. 1986). Although the study indicated that chum caught by purse net were larger than the purseseined pink salmon, no such species effect was present in the case where a beach seine was used (Fig. 2).

The patterns of fish body growth we observed were similar to those found in earlier studies (Jones \& Nemec 2004, Jones et al. 2006, Jones \& Hargreaves 2009). However, the detailed trends reported here provide a better understanding of factors influencing apparent fish size. For example, the apparently greater length of pink salmon in 2004 (shown in Fig. 2), appears to be an artefact of sampling effort as there were no fish samples from March (i.e. the smallest pink salmon) in 2004. Both fish species have similar patterns of apparent growth throughout the season, but chum appear to grow faster later in the year, consistent with the observations of the pink and chum population in Kamchatka (Karpenko \& Koval 2012) and in the Northern Bering and Chukchi Seas (Moss et al. 2009). There was little inter-annual variation in these growth patterns for either species over the study period. One exception was 2005, during which there was higher apparent growth for chum; though not an outlier year in pink salmon, growth was also higher in 2005 than most of the other observed years. Inter-annual variation in fish growth may be attributed to inter-annual variation in ocean hydrological factors affecting available food resources and fish metabolism (i.e. ectotherms grow faster in warmer temperatures; Gillooly et al. 2001, Atwood et al. 2012).

The inter-annual variation of sea lice infestation on juvenile chum and pink salmon was similar to those which have been observed in previous studies (Jones et al. 2006, Jones \& Hargreaves 2007, 2009, Saksida et al. 2011). With the exception of one year (2004), the prevalence of infestation appears to vary around the range 15 to $35 \%$ for both species. Both the descrip- 
Table 4. Lepeophtheirus salmonis and Caligus clemensi infesting Oncorhynchus keta and O. gorbuscha. Random effects logistic model for factors associated with the presence of sea lice on wild chum and pink salmon sampled by beach or purse seine in the Broughton Archipelago. Statistical significance at ${ }^{*} \mathrm{p}<0.01$

\begin{tabular}{|c|c|c|c|}
\hline Parameters & Coefficient $(\beta)$ & $95 \%$ CI of $\beta$ & Odds ratio \\
\hline \multicolumn{4}{|l|}{ Year* } \\
\hline 2003 & Reference & & \\
\hline 2004 & 1.46 & {$[1.40,1.51]$} & 4.30 \\
\hline 2005 & 0.06 & {$[-0.02,0.13]$} & 1.06 \\
\hline 2006 & -0.48 & {$[-0.54,-0.43]$} & 0.62 \\
\hline 2007 & -0.11 & {$[-0.16,-0.07]$} & 0.89 \\
\hline 2008 & -1.64 & {$[-1.69,-1.58]$} & 0.19 \\
\hline 2009 & -1.80 & {$[-1.86,-1.75]$} & 0.16 \\
\hline 2010 & -0.78 & {$[-0.86,-0.71]$} & 0.46 \\
\hline 2011 & -1.64 & {$[-1.73,-1.54]$} & 0.19 \\
\hline 2012 & -1.01 & {$[-1.08,-0.94]$} & 0.36 \\
\hline \multicolumn{4}{|l|}{ Month* } \\
\hline March & Reference & & \\
\hline April & 0.72 & {$[0.63,0.82]$} & 2.06 \\
\hline May & 1.02 & {$[0.92,1.12]$} & 2.77 \\
\hline June & 0.40 & {$[0.29,0.51]$} & 1.49 \\
\hline July & 0.13 & {$[-0.03,0.29]$} & 1.14 \\
\hline \multicolumn{4}{|l|}{ Gear type* } \\
\hline Beach seine & Reference & & \\
\hline Purse seine & -0.48 & {$[-0.54,-0.43]$} & 0.62 \\
\hline \multicolumn{4}{|l|}{ Fish species ${ }^{*}$} \\
\hline Chum & Reference & & \\
\hline Pink & -0.37 & {$[-0.401,-0.338]$} & \\
\hline Length* & 0.037 & {$[0.035,0.038]$} & \\
\hline Length $^{2 *}$ & -0.00058 & {$[-0.00062,-0.00055]$} & \\
\hline \multicolumn{4}{|l|}{ Interaction terms ${ }^{*}$} \\
\hline Fish species $\times$ Length ${ }^{*}$ & -0.016 & {$[-0.018,-0.014]$} & \\
\hline Fish species $\times$ Length $^{2 *}$ & 0.00024 & {$[0.00019,0.00030]$} & \\
\hline Intercept* & -1.46 & {$[-1.71,-1.21]$} & \\
\hline \multicolumn{4}{|l|}{ Random effect } \\
\hline Site*: variance (SE) & $2.04(0.27)$ & & \\
\hline Site-median odds-ratio & 3.90 & {$[4.50,12.27]$} & \\
\hline Intraclass correlation coefficier & ent 0.38 & & \\
\hline
\end{tabular}

tive summaries and the logistic model indicated a slightly higher level of infestation on chum than on pink salmon. However, fish size should also be taken into consideration, as pink salmon of 0.5 to $1.0 \mathrm{~g}$ are at greater risk of physiological compromise or mortality resulting from infestations with Lepeophtheirus salmonis (Jones \& Hargreaves 2009). Jones \& Hargreaves (2009) accounted for fish size using weight and found a decreasing monthly trend of L. Salmonis density (lice $\mathrm{g}^{-1}$ ) in pink salmon, which is similar to the result from this study using fish body length rather than weight.

The results also suggest that relative variation in prevalence and abundance are similar. At low to moderate prevalence levels, it can be shown theoretically that a linear relationship is likely to hold between prevalence and abundance due to the fact that a negative binomial distribution best describes the pattern of infestation (Shaw et al. 1998, Baillie et al. 2009). In our study, this linear relationship was clearly exhibited in all months other than those for which the prevalence was higher than $60 \%$. This has previously shown to be the case in empirical data from Scottish farms (Baillie et al. 2009) as well as for a much smaller sub-set of the data currently being analyzed, which looked only at infestation levels on wild pink salmon over a 4 yr period (Heuch et al. 2011).

Although our analytical approach could not account for sea lice species for most analyses given the lack of these data across the study, the relative proportions of the 2 major lice species on sampled fish was explored by computing the difference in proportion of Lepeophtheirus salmonis and Caligus clemensi to the total number of lice that were identified to species. We found no evidence of a difference in the distribution of lice species across the 2 host species. In the early years of the study, where L. salmonis was the dominant species, its higher relative proportion was seen on both host species. Likewise in later years, the trend shifted towards a more equal occurrence of both sea lice species or a predominance of C. clemensi (in 2011). Laboratory studies (Jones et al. 2007) suggested that juvenile chum salmon maintain higher burdens of L. salmonis compared to sizematched pink salmon. The data presented here on natural infestations do not contradict this finding; however, in a number of years and particularly early in the migration season, lice infestation levels appear to be similar across fish species.

A multivariable logistic regression helps increase our understanding of sea lice infestation on wild salmon over the past $10 \mathrm{yr}$ in the Broughton Archipelago by accounting for the possible confounding factors. The model supports the observation that temporal variation and spatial clustering exist in sea lice infestation of wild chum and pink juvenile salmon made in several studies (Jones \& Nemec 2004, Jones et al. 2006, Saksida et al. 2011). Moreover, the model 
indicated that the probability of lice infestation depends on fish length and that this risk factor differs between the 2 fish species studied. The probability followed a 'quadratic' pattern with likelihood of infestation increasing as fish grow until they reach a certain size, after which the likelihood of infestation decreases. The 'convex' pattern observed is one of a number of age-intensity relationships that have been proposed (Hudson \& Dobson 1995, Raffel et al. 2011) and would be consistent with a mechanism such as acquired immunity following prolonged exposure (Yang \& Yang 1998). Given that infestation is acquired after fish enter seawater, the initial increase in probability is not surprising. A number of mechanisms may explain the subsequent declining trend: (1) larger fish tend to be infested with older motile stage lice, and experimental data indicate few lice survive to reach motile stage (Jones et al. 2007, Krkošek et al. 2009); (2) larger fish may swim faster and have better developed scales, making it more difficult for copepodids to successfully make contact, attach, and survive (Tucker et al. 2002); or (3) infested fish may have been killed by direct and indirect effects of lice (Krkošek et al. 2011a). Additionally, larger fish tend to stay in deeper water, which has lower concentrations of copepodids than the shallow water near the shore (Costelloe et al. 1995, McKibben \& Hay 2004, Costello 2006). The finding that fish caught by purse seine have a lower likelihood of lice infestation may be confounded by the depth of the water, since purse seine is often used to catch fish in deep water, whereas beach seine is suitable for shallow water (Hahn et al. 2007). There was clear evidence as the season progressed and fish became larger that the sea lice became more mature. It is not known, however, why lice infestations on pink salmon later in the season were more likely in the form of motiles than those found on chum salmon.

This study provides a descriptive and comprehensive $10 \mathrm{yr}$ overview that merges for the first time data from 2 large monitoring programs of sea lice infestation on wild juvenile Pacific salmon in the Broughton Archipelago. These trends are consistent with trends observed over a shorter time frame from data limited to a more localized area (Tribune Channel) of the Broughton Archipelago (Peacock et al. 2013). There was inter-annual as well as inter-month variation around the prevalence of lice infestation on wild chum and pink salmon juveniles. While some differences were evident between infestation levels on the 2 host species, the overall prevalence followed similar trends, and this was true for both sea lice species, to the extent that this could be assessed. Further model- ing will be conducted to find biotic and abiotic factors which can better explain some of this variation, in particular the reasons why 2004 appears to show such marked difference from the rest of the decade. The model also indicated that around one-third of the variation in lice infestation was associated with unmeasured factors at the site level. This suggested that strong spatial clustering of sea lice infestation occurred in this area, consistent with localized sources of infestation and/or spatial aggregation of infested fish. Future studies will focus more directly on this spatial dimension as well as the environmental and aquaculture management factors which likely play an important role in sea lice infestation.

Acknowledgements. We thank members of the BAMP Science Team and sponsors who provided data and funding as well as valuable reviews and comments in support of the research summarized in this publication. The BAMP (www. bamp.ca) was initiated in 2010 as a multiyear sea lice monitoring and research program involving the federal government, salmon farm producers, conservationists, and academic researchers.

\section{LITERATURE CITED}

Atwood TB, Wiegner TN, MacKenzie RA (2012) Effects of hydrological forcing on the structure of a tropical estuarine food web. Oikos 121:277-289

Baillie M, Lees F, Gettinby G, Revie CW (2009) The use of prevalence as a measure of lice burden: a case study of Lepeophtheirus salmonis on Scottish Atlantic salmon, Salmo salar L., farms. J Fish Dis 32:15-25

Beamish RJ, Neville CM, Sweeting RM, Ambers N (2005) Sea lice on adult Pacific salmon in the coastal waters of Central British Columbia, Canada. Fish Res 76:198-208

$>$ Boxaspen K (2006) A review of the biology and genetics of sea lice. ICES J Mar Sci 63:1304-1316

Burnham KP (2002) Information and likelihood theory: a basis for model selection and inference. In: Burnham KP, Anderson DR (eds) Model selection and multi-model inference: a practical information-theoretic approach. Springer, New York, NY, p 49-97

Bush AO, Lafferty KD, Lotz JM, Shostak AW (1997) Parasitology meets ecology on its own terms: Margolis et al. revisited. J Parasitol 83:575-583

> Costello MJ (2006) Ecology of sea lice parasitic on farmed and wild fish. Trends Parasitol 22:475-483

Costelloe J, Costelloe M, Roche N (1995) Variation in sea lice infestation on Atlantic salmon smolts in Killary Harbour, West Coast of Ireland. Aquacult Int 3:379-393

Dawley EM, Ledgerwood RD, Blahm TH, Sims CW and others (1986) Migrational characteristics, biological observations, and relative survival of juvenile salmonids entering the Columbia River estuary, 1966-1983. US Department of Energy, Bonneville Power Administration, Division of Fish \& Wildlife, Portland, OR

Dohoo RI, Martin SW, Stryhn H (2009) Introduction to clustered data. In: McPike SM (ed) Veterinary epidemiologic research. VER, Inc., Charlottetown, p 529-552 
Gillooly JF, Brown JH, West GB, Savage VM, Charnov EL (2001) Effects of size and temperature on metabolic rate. Science 293:2248-2251

Gottesfeld AS, Proctor B, Rolston LD, Carr-Harris C (2009) Sea lice, Lepeophtheirus salmonis, transfer between wild sympatric adult and juvenile salmon on the north coast of British Columbia, Canada. J Fish Dis 32:45-57

Hahn PKJ, Bailey RE, Ritchie A (2007) Beach seining. In: Johnson DH, American Fisheries Society, State of the Salmon (Program) (eds) Salmonid field protocols handbook: techniques for assessing status and trends in salmon and trout populations. American Fisheries Society in association with State of the Salmon, Bethesda, MD, p 267-324

> Hamilton-West C, Arriagada G, Yatabe T, Valdés P, HervéClaude LP, Urcelay S (2012) Epidemiological description of the sea lice (Caligus rogercresseyi) situation in southern Chile in August 2007. Prev Vet Med 104:341-345

Heard WR (1991) Life history of pink salmon (Oncorhynchus gorbuscha). In: Groot C, Margolis L (eds) Pacific salmon life histories. UBC Press, Vancouver, p 119-230

> Heuch PA, Revie CW, Gettinby G (2003) A comparison of epidemiological patterns of salmon lice, Lepeophtheirus salmonis, infections on farmed Atlantic salmon, Salmo salar L., in Norway and Scotland. J Fish Dis 26:539-551

> Heuch PA, Gettinby G, Revie CW (2011) Counting sea lice on Atlantic salmon farms - empirical and theoretical observations. Aquaculture 320:149-153

Hudson PJ, Dobson A (1995) Macroparasites: observed patterns in naturally fluctuating animal populations. In: Grenfell BT, Dobson AP (eds) Ecology of infectious diseases in natural populations. Cambridge University Press, Cambridge, p 144-176

Jackson D, O' Donohoe P, Kane F, Kelly S and others (2012) Result of an epidemiological study of sea lice infestation in South Connemara, West of Ireland. Aquaculture 364365:118-123

Jacobsen JA, Gaard E (1997) Open-ocean infestation by salmon lice (Lepeophtheirus salmonis): comparison of wild and escaped farmed Atlantic salmon (Salmo salar L.). ICES J Mar Sci 54:1113-1119

Johnsen RD, Sims CW (1973) Purse seining for juvenile salmon and trout in the Columbia River estuary. Trans Am Fish Soc 102:341-345

Johnson SC, Fast MD (2004) Interactions between sea lice and their hosts. Symp Soc Exp Biol 55:131-159; Discussion 243-245

> Jones SRM, Hargreaves NB (2007) The abundance and distribution of Lepeophtheirus salmonis (Copepoda: Caligidae) on pink (Oncorhynchus gorbuscha) and chum (O. keta) salmon in coastal British Columbia. J Parasitol 93: 1324-1331

> Jones SRM, Hargreaves NB (2009) Infection threshold to estimate Lepeophtheirus salmonis-associated mortality among juvenile pink salmon. Dis Aquat Org 84: 131-137

Jones SRM, Nemec A (2004) Pink salmon action plan: sea lice on juvenile salmon and some non-salmonid species in the Broughton Archipelago in 2003. Canadian Science Advisory Secretariat Research Document 2004/105. Fisheries and Oceans Canada, Ottawa

Jones SRM, Wosniok W, Hargreaves NB (2006) The salmon louse Lepeophtheirus salmonis on salmonid and nonsalmonid fishes in British Columbia, Canada. In: Proc 11th Int Symp Vet Epidemiol Econ, Cairns, p 1131
Jones SRM, Fast MD, Johnson SC, Groman DB (2007) Differential rejection of salmon lice by pink and chum salmon: disease consequences and expression of proinflammatory genes. Dis Aquat Org 75:229-238

Karpenko VI, Koval MV (2012) Feeding strategies and trends of pink and chum salmon growth in the marine waters of Kamchatka. Tech Rep 8. North Pacific Anadromous Fish Commission, Vancouver, p 82-86

Krkošek M, Lewis MA, Volpe JP (2005a) Transmission dynamics of parasitic sea lice from farm to wild salmon. Proc R Soc Lond B Biol Sci 272:689-696

Krkošek M, Morton A, Volpe JP (2005b) Nonlethal assessment of juvenile pink and chum salmon for parasitic sea lice infections and fish health. Trans Am Fish Soc 134: 711-716

Krkošek M, Lewis MA, Morton A, Frazer LN, Volpe JP (2006) Epizootics of wild fish induced by farm fish. Proc Natl Acad Sci USA 103:15506-15510

Krkošek M, Ford JS, Morton A, Lele S, Myers RA, Lewis MA (2007a) Declining wild salmon populations in relation to parasites from farm salmon. Science 318:1772-1775

Krkošek M, Gottesfeld A, Proctor B, Rolston D, Carr-Harris C, Lewis MA (2007b) Effects of host migration, diversity and aquaculture on sea lice threats to Pacific salmon populations. Proc R Soc Lond B Biol Sci 274:3141-3149

Krkošek M, Morton A, Volpe JP, Lewis MA (2009) Sea lice and salmon population dynamics: effects of exposure time for migratory fish. Proc R Soc Lond B Biol Sci 276: 2819-2828

Krkošek M, Connors BM, Ford H, Peacock S and others (2011a) Fish farms, parasites, and predators: implications for salmon population dynamics. Ecol Appl 21:897-914

Krkošek M, Connors BM, Morton A, Lewis MA, Dill LM, Hilborn R (2011b) Effects of parasites from salmon farms on productivity of wild salmon. Proc Natl Acad Sci USA 108:14700-14704

> Marty GD, Saksida SM, Quinn TJ (2010) Relationship of farm salmon, sea lice, and wild salmon populations. Proc Natl Acad Sci USA 107:22599-22604

> McKibben MA, Hay DW (2004) Distributions of planktonic sea lice larvae Lepeophtheirus salmonis in the inter-tidal zone in Loch Torridon, Western Scotland in relation to salmon farm production cycles. Aquacult Res 35:742-750

Morton AB, Williams R (2003) First report of a sea louse, Lepeophtheirus salmonis, infestation on juvenile pink salmon, Oncorhynchus gorbuscha, in nearshore habitat. Can Field Nat 117:634-641

> Morton A, Routledge RD, Williams R (2005) Temporal patterns of sea louse infestation on wild Pacific salmon in relation to the fallowing of Atlantic salmon farms. N Am J Fish Manag 25:811-821

> Morton A, Routledge R, Krkošek M (2008) Sea louse infestation in wild juvenile salmon and Pacific herring associated with fish farms off the east-central coast of Vancouver Island, British Columbia. N Am J Fish Manag 28: $523-532$

Moss JH, Murphy JM, Farley EV, Eisner LB, Andrews AG (2009) Juvenile pink and chum salmon distribution, diet, and growth in the northern Bering and Chukchi seas. North Pac Anadromous Fish Comm Bull 5:191-196

Mustafa A (1997) Host factors important in determining infection intensity with the sea louse, Caligus elongatus Nordmann, 1832, in Atlantic salmon, Salmo salar L. and Arctic charr, Salvelinus alpinus (L.). PhD dissertation, University of New Brunswick, Fredericton 
Nendick L, Sackville M, Tang S, Brauner CJ, Farrell AP (2011) Sea lice infection of juvenile pink salmon (Oncorhynchus gorbuscha): effects on swimming performance and postexercise ion balance. Can J Fish Aquat Sci 68: 241-249

Noakes DJ, Beamish RJ (2011) Shifting the balance: towards sustainable salmon populations and fisheries of the future. In: Taylor WW, Lynch AJ, Schechter MG (eds) Sustainable fisheries: multi-level approaches to a global problem. American Fisheries Society, Bethesda, MD, p 23-50

Peacock SJ, Krkošek M, Proboszcz S, Orr C, Lewis MA (2013) Cessation of a salmon decline with control of parasites. Ecol Appl 23:606-620

Pike AW, Wadsworth SL (1999) Sealice on salmonids: their biology and control. Adv Parasitol 44:233-337

Price MHH, Morton A, Reynolds JD (2010) Evidence of farm-induced parasite infestations on wild juvenile salmon in multiple regions of coastal British Columbia, Canada. Can J Fish Aquat Sci 67:1925-1932

Price MHH, Proboszcz SL, Routledge RD, Gottesfeld AS, Orr C, Reynolds JD (2011) Sea louse infection of juvenile sockeye salmon in relation to marine salmon farms on Canada's west coast. PLoS ONE 6:e16851

Raffel TR, Lloyd-Smith JO, Sessions SK, Hudson PJ, Rohr JR (2011) Does the early frog catch the worm? Disentangling potential drivers of a parasite age-intensity relationship in tadpoles. Oecologia 165:1031-1042

Rolston D, Proctor B (2003) Salmon farms and sea lice: a baseline report on the incidence of sea lice on juvenile salmonids on British Columbia's North Coast. David Suzuki Foundation, Vancouver

Saksida SM, Greba L, Morrison D, Revie CW (2011) Sea lice on wild juvenile Pacific salmon and farmed Atlantic

Editorial responsibility: David Marcogliese, Montreal, Quebec, Canada salmon in the northernmost salmon farming region of British Columbia. Aquaculture 320:193-198

Salo EO (1991) Life history of chum salmon (Oncorhynchus keta). In: Groot C, Margolis L (eds) Pacific salmon life histories. UBC Press, Vancouver, p 231-309

Shaw DJ, Grenfell BT, Dobson AP (1998) Patterns of macroparasite aggregation in wildlife host populations. Parasitology 117:597-610

Sims CW, Johnsen RC (1974) Variable-mesh beach seine for sampling juvenile salmon in Columbia River estuary. Mar Fish Rev 36:23-26

> Tucker CS, Sommerville C, Wootten R (2002) Does size really matter? Effects of fish surface area on the settlement and initial survival of Lepeophtheirus salmonis, an ectoparasite of Atlantic salmon Salmo salar. Dis Aquat Org 49:145-152

$>$ Tully O, Nolan DT (2002) A review of the population biology and host-parasite interactions of the sea louse Lepeophtheirus salmonis (Copepoda: Caligidae). Parasitology 124(Suppl):S165-S182

Wagner GN, Fast MD, Johnson SC (2008) Physiology and immunology of Lepeophtheirus salmonis infections of salmonids. Trends Parasitol 24:176-183

Wertheimer AC, Fergusson EA, Focht RL, Heard WR, Orsi JA, Sturdevant MV, Wing BL (2003) Sea lice infection of juvenile salmon in the marine waters of the northern region of southeastern Alaska, May-August, 2003. NPAFC Doc 706. Auke Bay Laboratory, Alaska Fisheries Science Center, NMFS, NOAA, US Department of Commerce, Juneau, AK, p 1-13

Yang HM, Yang AC (1998) The stabilizing effects of the acquired immunity on the schistosomiasis transmission modeling - the sensitivity analysis. Mem Inst Oswaldo Cruz 93(Suppl 1):63-73

Submitted: December 27, 2012; Accepted: May 3, 2013 Proofs received from author(s): July 6, 2013 